\title{
Dysphagia rehabilitation following stroke: more than just functional recovery
}

Moloney, J. ${ }^{1}$ \& Walshe, M. ${ }^{2}$

1. PhD Candidate, Department of Clinical Speech and Language Studies, Trinity College Dublin

2. Associate Professor, Head of Department, Department of Clinical Speech and Language Studies, Trinity College Dublin

\section{OVERVIEW}

\section{Background:}

The Burden of Stroke in Europe Report (King's College London, 2017) estimates that the number of people living with stroke related disability across Europe is likely to rise by $25 \%$ by the year 2035. With this ever increasing prevalence, the need for evidence based, comprehensive and patient-centred approaches to dysphagia rehabilitation will be greater than ever before.

Therapeutic interventions targeting physical recovery in swallow function continue to be the primary focus for Speech and Language Therapists (SLT) and appropriately so. However, a growing evidence base is consistently demonstrating the significant psychosocial impact of dysphagia in stroke (Moloney \& Walshe, 2017; Klinke et al, 2013; Medin et al, 2010).

Accordingly, best practice guidelines in the management of dysphagia following stroke recommend that healthcare professionals should be aware of the potential psychosocial impact of dysphagia and should address any quality of life (QOL) concerns which may arise (SIGN, 2010).

\section{Research Questions:}

Considering the above, the specific research questions of the current project were

1. What are the current practices of SLTs internationally when addressing QOL in the management of dysphagia in stroke?

2. What are the beliefs of SLTs internationally regarding the impact of dysphagia on QOL in persons following stroke?

3. What are the current barriers and facilitators to practice in the assessment and management of QOL in dysphagia following stroke?

\section{Methods:}

An anonymous cross-sectional non-experimental survey study design was used. The 18 question survey, consisting of both open and closed questions and designed using Survey Monkey, was disseminated internationally through professional bodies and academic institutions. Purposive and snowball sampling was used. Participants were professionally qualified SLTs with sufficient use of the English language to complete the survey.

\section{FINDINGS}

\section{Participants:}

223 participants across 20 different countries including Ireland, the UK, South Africa, Australia, New Zealand, Canada, United States, Brazil, Singapore, Saudi Arabia, Malta, and mainland Europe took part.

As can be seen in the graph below, the majority of these participants work in an acute hospital setting.

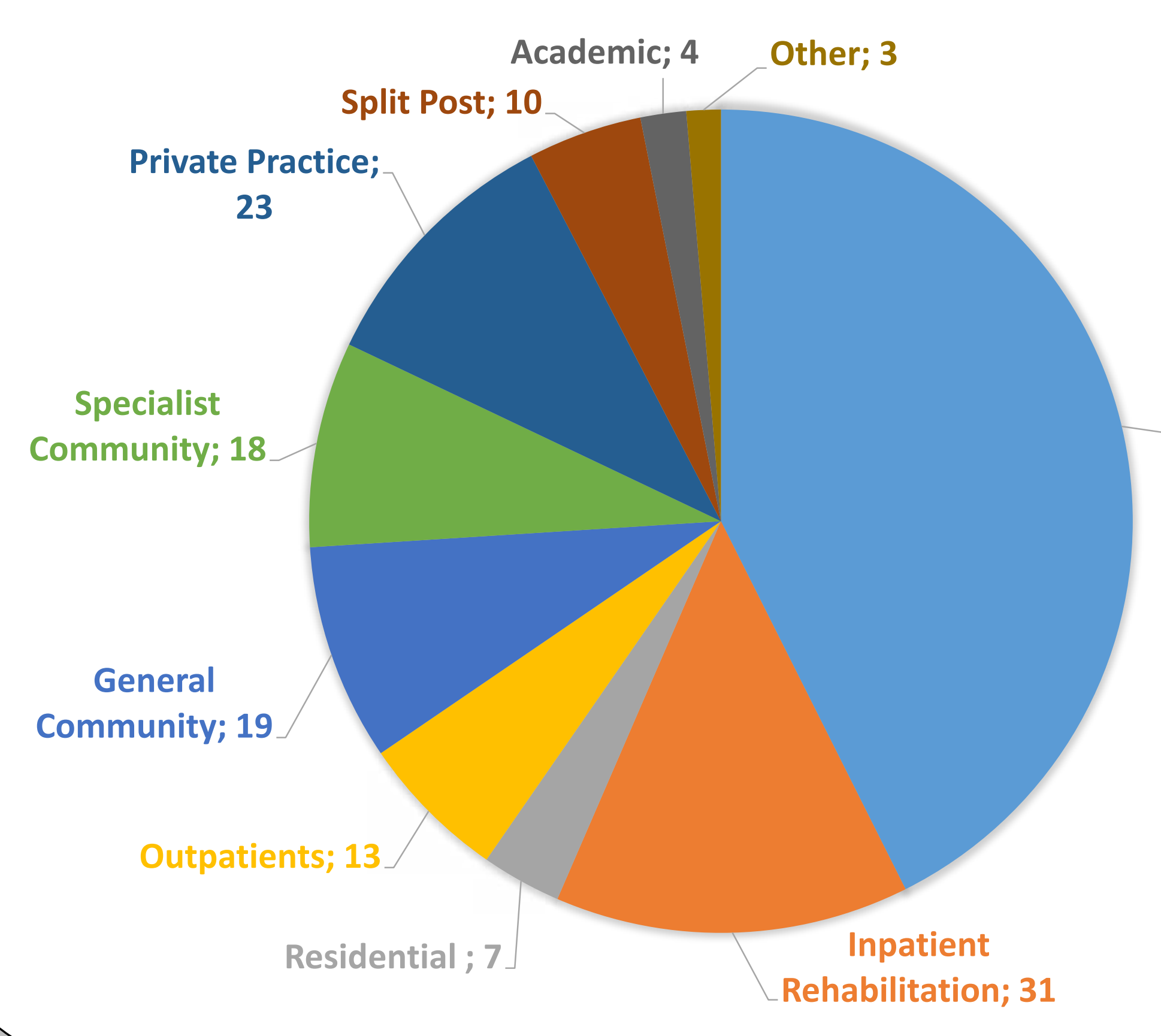

\section{Current Practice and Beliefs:}

- Over $85 \%$ of participants believe dysphagia will have a significant impact on QOL

- Participants believe persons with dysphagia related to progressive neurological conditions and head and neck cancer are more likely to experience poor QOL than persons with dysphagia as a result of stroke.

- Only $42 \%$ of participants indicated that they fee knowledgeable regarding $\mathrm{QOL}$ issues in dysphagia in the stroke population.

- Participants are most likely to discontinue direct rehabilitation after 3-6 months if little or no progress in swallowing function has been made.

- Although participants believe QOL should then become the primary goal of care, over $60 \%$ report they would discharge the person with dysphagia from their caseload at this point.

- Education and training was not highlighted as a primary goal of care by any participants in any stage of the person's rehabilitation journey. 